\title{
Quintic B-spline collocation method for numerical solution of free vibration of tapered Euler-Bernoulli beam on variable Winkler foundation
}

\author{
A. Ghannadiasl \\ Faculty Department of Civil Engineering, Faculty of Engineering, University of Mohaghegh Ardabili, Ardabil, Iran \\ Phone: +989144511813; Fax: +984531505720
}

ABSTRACT - The collocation method is the method for the numerical solution of integral equations and partial and ordinary differential equations. The main idea of this method is to choose a number of points in the domain and a finite-dimensional space of candidate solutions. So, that solution satisfies the governing equation at the collocation points. The current paper involves developing, and a comprehensive, step-by step procedure for applying the collocation method to the numerical solution of free vibration of tapered Euler-Bernoulli beam. In this stusy, it is assumed the beam rested on variable Winkler foundation. The simplicity of this approximation method makes it an ideal candidate for computer implementation. Finally, the numerical examples are introduced to show efficiency and applicability of quintic B-spline collocation method. Numerical results are demonstrated that quintic B-spline collocation method is very competitive for numerical solution of frequency analysis of tapered beam on variable elastic foundation.

\author{
ARTICLE HISTORY \\ Received: $07^{\text {th }}$ May 2020 \\ Revised: 29th Dec 2020 \\ Accepted: 29th Jan 2021 \\ KEYWORDS \\ Collocation method; \\ Tapered Euler-Bernoulli \\ beam; \\ Winkler foundation; \\ boundary conditions; \\ B-spline function
}

\section{INTRODUCTION}

Many engineering problems can be idealized as a beam on foundation. Winkler, Kerr, Pasternak, Viscoelastic, Vlasov and Hetenyi models are different types of foundation that can be used in modelling foundation in these problems [1]. The most common model is Winkler foundation. However, the Winkler model is inadequate for modelling of soil in various problems [2]. The quintic B-spline collocation method is used to numerical solution of free vibration of tapered damped Euler-Bernoulli (EB) beam on foundation in this paper. A spline function is a piecewise polynomial function in a variable $\mathrm{x}$. The spline function is the composite of several internal point that number points must greater than or equal to $(k+1)$ degree. The B-spline functions of $(k+1)$ degree is used to solve the differential equation with $(k)$ degree [3]. Also, highest grade of B-spline function recursively comes of B-spline functions with lower grade.

Over the years, collocation method is applied to solve differential equations with different boundary conditions. An overview of the formulation, analysis and implementation of orthogonal spline collocation is provided for numerical solution of differential equations in two space variables by Bialecki and Fairweather [4]. The sextic B-spline function for numerical solution of a system of second-order boundary value problems is presented by Rashidinia et al. [5]. In this paper, the results are compared with the finite difference method (FDM) and it is demonstrated the results using the sextic B-spline collocation method are better than the FDM. The quartic B-spline collocation method is applied for numerical solution of Burgers' equation by Saka and Dağ [6]. Also, two-parameter singularly perturbed boundary value problem is solved using the B-spline method by Kadalbajoo and Yadaw [7]. In this paper, it is shown that the convergence analysis is a uniform convergence of second order. Quintic nonpolynomial B-spline collocation for a fourth-order boundary value problem is investigated by Ramadan et al. [8]. The results are shown that the quintic nonpolynomial B-spline collocation method presents better approximations. On the other hand, the presented method generalized all existing polynomial Bspline methods up to fourth-order.

Hsu applied B-spline collocation method for estimated the free vibration of non-uniform EB with typical boundary conditions on a uniform foundation [9]. The boundary conditions that accompanied the spline collocation method are used to convert the partial differential equations of non-uniform EB vibration problem into a discrete eigenvalue problem. Aziz and Šarler proposed the uniform Haar wavelets for the second-order boundary-value problems [10]. The isogeometric collocation method is presented for analysis of Timoshenko beam by Da Veiga et al. [11]. Zarebnia and Parvaz solved the Kuramoto-Sivashinsky equation using septic B-spline collocation method [12]. A linear combination of these functions is used to approximate solution. In this paper, using the Von-Neumann stability analysis technique, it is shown that the septic B-spline collocation method is unconditionally stable. Also, cubic B-spline collocation method is used to find the solution of the problem arising from chemical reactor theory [13]. The sextic B-spline collocation method is applied to find the numerical solution of the problem with the partial differential equation by Mohammadi [14]. The convergence analysis for present approximation is explored in details for EB with cantilever and fixed boundary conditions. The isogeometric collocation method is applied to solution of thin structural problems that describe using the EB and Kirchhoff plate models by Reali and Gomez [15]. Also, the isogeometric method is used for numerical solution 
of plate problems that describe using Reissner-Mindlin plate model by Kiendl et al. [16]. On the other hand, Akram applied the sextic B-spline collocation method for solving of boundary value problems with fifth-order [17]. The numerical results are shown that the presented method developed is better than quartic spline method. The isogeometric collocation method is used to analysis of spatial rods by Auricchio et al. [18]. The convergence and stability analysis are shown using the theoretical analysis in this paper. Liu and Li applied the energetic boundary functions collocation method for composite beams [19]. Also, the approximate solution based on collocation method is presented for the boundary value problem [20]. Chebyshev wavelet collocation method is used by Çelik for the non-uniform Euler-Bernoulli beam [21]. In this study, the beam is assumed with various supporting conditions. Kiendl et al. develop the isogeometric collocation method for the Timoshenko beam [22]. Also, frequancy analysis of graded tapered beam is presented using chebyshev collocation method by Chen [23].

In this study, analysis of elastically restrained tapered EB on variable Winkler foundation is presented using quintic B-spline collocation method. In the other hand, a damped EB on variable Winkler foundation is presented in a general form. In this paper, the main objective is to introduce a practical numerical solution based on quintic B-spline collocation method for elastically restrained tapered damped EB rested on variable Winkler foundation. For this propose, in section 2 , the quintic B-spline collocation method outlines. Then, the presented method is applied to the frequancy analysis of tapered damped EB rested on the variable Winkler foundation in section 3. So, section 4 explains various numerical examples to display applicability and efficiency of presented method. Finally, conclusions are introduced in section 5 , briefly.

\section{QUINTIC B-SPLINE COLLOCATION METHOD}

Let $x=\left(x_{0}, x_{1}, \ldots, x_{N}\right)$ be knot vector. The $k$ degree B-spline function can be given as follows [24]:

$$
B_{k, i}(x)=\frac{x-x_{i}}{x_{(i+k)}-x_{i}} B_{(k-1), i}(x)+\frac{x_{(i+k+1)}-x}{x_{(i+k+1)}-x_{(i+1)}} B_{(k-1),(i+1)}(x)
$$

and for $k=0$, the $\mathrm{B}$-spline function is determined as follow:

$$
B_{0, i}= \begin{cases}1 & \text { for } x \in\left[x_{i}, x_{(i+1)}\right) \\ 0 & \text { otherwise }\end{cases}
$$

where $0 £ i £ N-k-1$ and $1 £ k £ N-1$. Therefore, the quintic B-spline function can be given using Eq. (1). The quintic B-spline function, $B_{5, i}(x)$ is as follows:

$$
\begin{aligned}
& B_{i}(x) \\
& =\frac{1}{120 h^{5}} \begin{cases}\left(x-x_{i}+3 h\right)^{5} & x \in\left[x_{(i-3)}, x_{(i-2)}\right) \\
\left(x-x_{i}+3 h\right)^{5}-6\left(x-x_{i}+2 h\right)^{5} & x \in\left[x_{(i-2)}, x_{(i-1)}\right) \\
\left(x-x_{i}+3 h\right)^{5}-6\left(x-x_{i}+2 h\right)^{5}+15\left(x-x_{i}+h\right)^{5} & x \in\left[x_{(i-1)}, x_{i}\right) \\
\left(-x+x_{i}+3 h\right)^{5}-6\left(-x+x_{i}+2 h\right)^{5}+15\left(-x+x_{i}+h\right)^{5} & x \in\left[x_{i}, x_{(i+1)}\right) \\
\left(-x+x_{i}+3 h\right)^{5}-6\left(-x+x_{i}+2 h\right)^{5} & x \in\left[x_{(i+1)}, x_{(i+2)}\right) \\
\left(-x+x_{i}+3 h\right)^{5} & x \in\left[x_{(i+2)}, x_{(i+3)}\right) \\
0 & \text { otherwise }\end{cases}
\end{aligned}
$$

The solution domain is $0 £ x £ L$ in this paper. This domain is divided into $\mathrm{N}$ segments with length $h=L / N$, by the knots $x_{i}$ where $i=0,1, \ldots, N$ and $0=x_{0}<x_{1}<\ldots<x_{N}=L$. In quintic B-spline collocation method, basic function is defined as follows:

$$
y(x)=\sum_{i=-2}^{N+2} c_{i} B_{i}(x)
$$

where $B_{-2}(x), \ldots, B_{N+2}(x)$ are the quintic B-spline functions at knots. Also, $c_{-2}, \ldots, c_{N+2}$ are unknown coefficients that can be determined using the collocation form of the governing differential equation of the tapered damped EB rested on variable Winkler foundation and boundary conditions at each end of the beam. On the other hand, $1^{\text {st }}, 2^{\text {nd }}, 3^{\text {rd }}$ and $4^{\text {td }}$ 
derivatives of $B_{i}(x)$ respect to variable $x$ may be used in the governing differential equation of the EB. Table 1 is presented the values of $B_{i}(x)$ and its derivatives at the nodal points.

\section{MODELLING OF TAPERED DAMPED EB ON FOUNDATION}

A tapered damped EB on variable Winkler foundation is considered in this paper. This beam is restrained against rotation and translation at its ends, as shown in Figure $1 . K_{R L}$ and $K_{R R}$ are rotational coefficients at left and right edges, respectively. Also, $\mathrm{K}_{\mathrm{TL}}$ and $\mathrm{K}_{\mathrm{TR}}$ are transverse coefficients at left and right edges, respectively. For the free vibration of the tapered damped EB on variable Winkler foundation, the governing differential equation can be given by:

$$
\left(E I W_{, x x x x}+2 E I_{, x} W_{, x x x}+E I_{, x x} W_{, x x}\right)+\left(r_{i} W_{, x x x x t}+2 r_{i, x} W_{, x x x t}+r_{i, x x} W_{, x x t}\right)+\rho A W_{, t t}+r_{e} W_{, t}+K_{f} W=0
$$

where $\mathrm{W}$ is transverse deflection of EB, $A(x)$ and $I(x)$ denote the cross section function and moment of inertia function (at $x$ position), respectively. Also, $E, r_{i}, r_{e}$ and $\rho$ present the Young's modulus, internal damping coefficient of damped EB, it is generally very small [25], the external damping coefficient of damped EB, and material density, respectively. In this paper, it is assumed that the Winkler foundation modulus $\left(K_{f}(x)\right)$ through the EB length can vary constantly or linearly. Therefore, the $K_{f}(x)$ is given below:

$$
K_{f}(x)=K_{f 0}(1-\beta x)
$$

where $K_{f 0}$ is the foundation modulus at $x=0$ and $\beta$ is the variation parameters. The damping effects are assumed to be proportional to the stiffness properties of beam for internal damping and mass of beam for external damping in this study, respectively. Therefore, these damping can be considered as [26]:

$$
\begin{aligned}
& r_{i}(x)=\alpha_{i} E I(x) \\
& r_{e}(x)=\alpha_{e} \rho A(x)
\end{aligned}
$$

where in these equation, $\alpha_{i}$ and $\alpha_{e}$ are proportionality constants. On the other hand, the dimensionless damping ratio of damped EB with internal and external damping can be represented as (for $n^{\text {th }}$ mode):

Table 1. $B_{i}(x)$ and its derivatives at nodal points

\begin{tabular}{cccccccc}
\hline & $x_{i-3}$ & $x_{i-2}$ & $x_{i-1}$ & $x_{i}$ & $x_{i+1}$ & $x_{i+2}$ & $x_{i+3}$ \\
\hline$B_{i}$ & 0 & $\frac{1}{120}$ & $\frac{26}{120}$ & $\frac{66}{120}$ & $\frac{26}{120}$ & $\frac{1}{120}$ & 0 \\
$h B_{i}^{\prime}$ & 0 & $\frac{5}{120}$ & $\frac{50}{120}$ & 0 & $-\frac{50}{120}$ & $-\frac{5}{120}$ & 0 \\
$h^{2} B_{i}^{\prime \prime}$ & 0 & $\frac{20}{120}$ & $\frac{40}{120}$ & -1 & $\frac{40}{120}$ & $\frac{20}{120}$ & 0 \\
$h^{3} B_{i}^{\prime \prime \prime}$ & 0 & $\frac{60}{120}$ & -1 & 0 & 1 & $-\frac{60}{120}$ & 0 \\
$h^{5} B_{i}^{(4)}$ & 0 & 1 & -4 & 6 & -4 & 1 & 0 \\
\hline
\end{tabular}




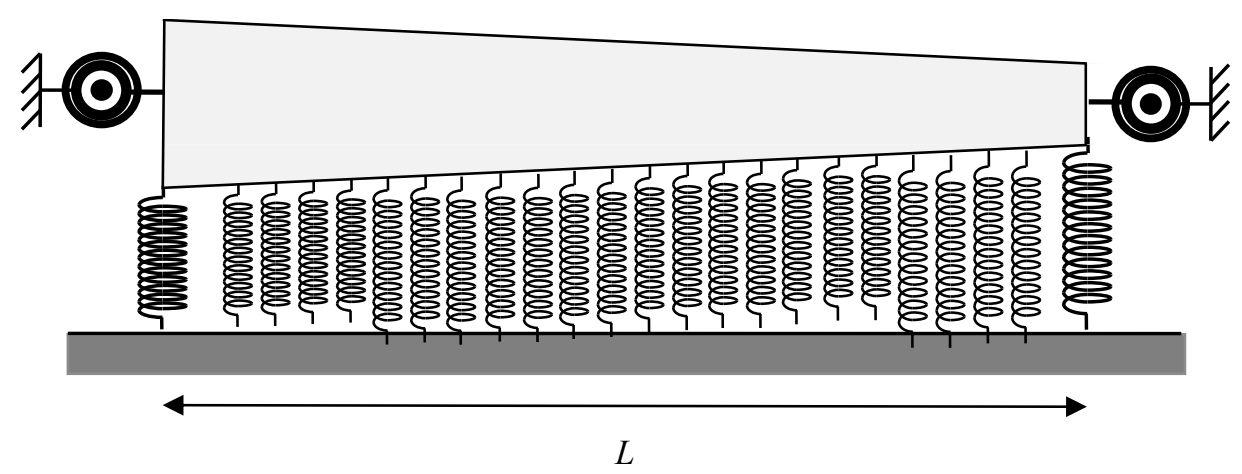

Figure 1. Tapered damped EB with general boundary conditions resting on Winkler foundation

$$
\begin{gathered}
\xi_{i}=\frac{1}{2} \alpha_{i} \omega_{n} \\
\xi_{e}=\frac{1}{2} \frac{\alpha_{e}}{\omega_{n}}
\end{gathered}
$$

The transverse deflection of EB can be considered as:

$$
W(x, t)=w(x) \operatorname{Exp}(i \omega t)
$$

where $\omega$ is circular frequency of tapered EB. In addition, $w(x)$ is deflection amplitude of the beam. So, by substituting Eq. (11) into Eq. (5), the governing differential equation result as:

$$
\left(1+\mathrm{i} \alpha_{i} \omega\right)\left(E I W_{, x x x x}+2 E I_{, x} W_{, x x x}+E I_{, x x} W_{, x x}\right)+\left(K_{f}-\rho \omega\left(\omega-\mathrm{i} \alpha_{e}\right) A\right) W=0
$$

Based on EB theory, general boundary conditions are given below [22]:

$$
\begin{array}{ll}
M(0, t)=K_{R L} \theta(0, t) & V(0, t)=-K_{T L} W(0, t) \\
M(L, t)=-K_{R R} \theta(L, t) & V(L, t)=K_{T R} W(L, t)
\end{array}
$$

where $M, V$ and $\theta$ are the bending moment $\left(M=E I \frac{\partial^{2} W}{\partial x^{2}}\right)$, the shear force $\left(\theta=\frac{\partial}{\partial x}\left(E I \frac{\partial^{2} W}{\partial x^{2}}\right)\right.$, and the slope ( $\theta=\frac{\partial W}{\partial x}$ ), respectively. Standard boundary conditions for EB theory are presented in Table 2. For this study, quintic Bspline collocation method is used to analyze tarped damped EB. By applying quintic B-spline function, Eq. (12) can be written as the following for $j=0,1, \ldots, N$ :

$$
\begin{array}{r}
\left(1+\mathrm{i} \alpha_{i} \omega\right)\left(E I\left(x_{j}\right) \sum_{i=-2}^{N+2} c_{i} B_{i}^{(4)}\left(x_{j}\right)+2 E I^{\prime}\left(x_{j}\right) \sum_{i=-2}^{N+2} c_{i} B_{i}^{(3)}(x)+E I^{\prime \prime}\left(x_{j}\right) \sum_{i=-2}^{N+2} c_{i} B_{i}^{(2)}\left(x_{j}\right)\right)+ \\
\left(K_{f}\left(x_{j}\right)-\rho \omega\left(\omega-\mathrm{i} \alpha_{e}\right) A\left(x_{j}\right)\right) \sum_{i=-2}^{N+2} c_{i} B_{i}\left(x_{j}\right)=0
\end{array}
$$

From Table 1 and Eq. (4), $y_{i}, y_{i}^{\prime}, y_{i}^{\prime \prime}, y_{i}^{\prime \prime \prime}$, and $y_{i}^{(4)}$ at node points are obtained as follows: 
Table 2. Standard boundary conditions for EB theory

\begin{tabular}{ll}
\hline Support & Boundary conditions \\
\hline Free & $\frac{\partial^{2} W}{\partial x^{2}}=\frac{\partial}{\partial x}\left(E I \frac{\partial^{2} W}{\partial x^{2}}\right)=0$ \\
Simply supported & $W=\frac{\partial^{2} W}{\partial x^{2}}=0$ \\
Sliding & $\frac{\partial W}{\partial x}=\frac{\partial}{\partial x}\left(E I \frac{\partial^{2} W}{\partial x^{2}}\right)=0$ \\
Clamped & $W=\frac{\partial W}{\partial x}=0$ \\
\hline
\end{tabular}

$$
\begin{gathered}
y_{i}=\frac{1}{120}\left(c_{i-2}+26 c_{i-1}+66 c_{i}+26 c_{i+1}+c_{i+2}\right) \\
y_{i}^{\prime}=\frac{1}{120 h}\left(5 c_{i-2}+50 c_{i-1}-50 c_{i+1}-5 c_{i+2}\right) \\
y_{i}^{\prime \prime}=\frac{1}{120 h^{2}}\left(20 c_{i-2}+40 c_{i-1}-120 c_{i}+40 c_{i+1}+20 c_{i+2}\right) \\
y_{i}^{\prime \prime \prime}=\frac{1}{120 h^{3}}\left(60 c_{i-2}-120 c_{i-1}+120 c_{i+1}-60 c_{i+2}\right) \\
y_{i}^{(4)}=\frac{1}{120 h^{4}}\left(120 c_{i-2}-480 c_{i-1}+720 c_{i}-480 c_{i+1}+120 c_{i+2}\right)
\end{gathered}
$$

Therefore, Eq. (14) can be stated as for $j=0,1, \ldots, N$ :

$$
\begin{aligned}
& \left(20 \lambda_{1}\left(6 I\left(x_{j}\right)+6 h I^{\prime}\left(x_{j}\right)+h^{2} I^{\prime \prime}\left(x_{j}\right)\right)+\left(K_{f}\left(x_{j}\right)-\lambda_{2} A\left(x_{j}\right)\right)\right) c_{i-2}+ \\
& \left(40 \lambda_{1}\left(-12 I\left(x_{j}\right)-6 h I^{\prime}\left(x_{j}\right)+h^{2} I^{\prime \prime}\left(x_{j}\right)\right)+26\left(K_{f}\left(x_{j}\right)-\lambda_{2} A\left(x_{j}\right)\right) \lambda_{2}\right) c_{i-1}+ \\
& \left(120 \lambda_{1}\left(6 I\left(x_{j}\right)-h^{2} I^{\prime \prime}\left(x_{j}\right)\right)+66\left(K_{f}\left(x_{j}\right)-\lambda_{2} A\left(x_{j}\right)\right) \lambda_{2}\right) c_{i}+ \\
& \left(40 \lambda_{1}\left(-12 I\left(x_{j}\right)+6 h I^{\prime}\left(x_{j}\right)+h^{2} I^{\prime \prime}\left(x_{j}\right)\right)+26\left(K_{f}\left(x_{j}\right)-\lambda_{2} A\left(x_{j}\right)\right) \lambda_{2}\right) c_{i+1}+ \\
& \left(20 \lambda_{1}\left(6 I\left(x_{j}\right)-6 h I^{\prime}\left(x_{j}\right)+h^{2} I^{\prime \prime}\left(x_{j}\right)\right)+\left(K_{f}\left(x_{j}\right)-\lambda_{2} A\left(x_{j}\right)\right) \lambda_{2}\right) c_{i+2}=0
\end{aligned}
$$

where:

$$
\begin{aligned}
& \lambda_{1}=\frac{E}{h^{4}}\left(1+\mathrm{i} \alpha_{i} \omega\right) \\
& \lambda_{2}=\rho \omega\left(\omega-\mathrm{i} \alpha_{e}\right)
\end{aligned}
$$

The system in Eq. (16) is the set of $N+1$ equations with $N+5$ unknowns. The boundary conditions at each end present four extra equations. Thus, these equations depend on end support. For example, these equations for simply supported condition can be given as:

$$
\begin{gathered}
c_{-2}+26 c_{-1}+66 c_{0}+26 c_{1}+c_{2}=0 \\
c_{-2}+2 c_{-1}-6 c_{0}+2 c_{1}+c_{2}=0 \\
c_{N-2}+2 c_{N-1}-6 c_{N}+2 c_{N+1}+c_{N+2}=0 \\
c_{N-2}+26 c_{N-1}+66 c_{N}+26 c_{N+1}+c_{N+2}=0
\end{gathered}
$$


Finally, N+5 algebraic equations are obtained. $c_{-2}, c_{-1}, \ldots, c_{N+1}, c_{N+2}$ and $\omega$ are the unknown variables in these algebraic equations. Therefore, the matrix equation is given as:

$$
\left[\begin{array}{cccccc}
A_{-2,-2} & A_{-2,-1} & A_{-2,0} & \cdot & \cdot & A_{-2, N+2} \\
A_{-1,-2} & A_{-1,-1} & A_{-1,0} & \cdot & \cdot & A_{-1, N+2} \\
A_{0,-2} & A_{0,-1} & A_{0,0} & \cdot & \cdot & A_{0, N+2} \\
\cdot & \cdot & \cdot & \cdot & \cdot & \cdot \\
\cdot & \cdot & \cdot & \cdot & \cdot & \cdot \\
A_{N+2,-2} & A_{N+2,-1} & A_{N+2,0} & \cdot & \cdot & A_{N+2, N+2}
\end{array}\right]\left[\begin{array}{c}
c_{-2} \\
c_{-1} \\
c_{0} \\
\cdot \\
\cdot \\
c_{N+2}
\end{array}\right]=\left[\begin{array}{c}
0 \\
0 \\
0 \\
\cdot \\
\cdot \\
0
\end{array}\right]
$$

In matrix Eq. (18), the nontrivial solution, when determinant of coefficients is equal to zero, is acquired. The coefficients matrix determinant is the associated frequency equation. In this paper, the numerical solution for frequency analysis of tapered damped EB that is generated using proposed method has a general form.

\section{NUMERICAL RESULTS}

To evaluate efficiency of proposed method, it is applied for solving the some examples. Also, the numerical computations are performed by the WOLFRAM MATHEMATICA software.

\section{Uniform EB with Standard Boundary Conditions}

To demonstrate accuracy of presented solution, an uniform EB is assumed with standard boundary conditions. The frequency parameters $\left(\varphi=\omega \sqrt{\rho A L^{4} / E I}\right.$ ) of the uniform EBs using the presented method along with the power series method [27] and the exact solution [28] and are compared in Table 3. Results are presented that the maximum difference is $1.74 \%$ for $\mathrm{N}=25$ and $0.44 \%$ for $\mathrm{N}=50$, hence, they are fairly close. Convergence of first five frequencies are demonstrated in Figure 2. The first natural frequancy of the uniform EB is less sensitive to number of terms. In addition, the maximum difference of the frequency parameter for clamped-free EB is approximately $12.41 \%$ for $\mathrm{N}=5$ and $0.19 \%$ for $\mathrm{N}=60$.

\section{Cantilever Tapered EB on Uniform Winkler Foundation}

In this example, effect of elastic Winkler foundation on frequency parameters of the cantilever tapered EB is presented. The tapered beam characteristics is considered as:

$$
A(x)=A_{0}\left(1-0.5 \frac{x}{L}\right) \quad I(x)=I_{0}\left(1-0.5 \frac{x}{L}\right)^{3}
$$

\begin{tabular}{|c|c|c|c|c|c|c|c|}
\hline \multirow[b]{2}{*}{ Supported } & \multirow{2}{*}{$\frac{0}{8}$} & \multirow{2}{*}{$\begin{array}{c}\text { Exact } \\
\text { Solution } \\
\text { [28] }\end{array}$} & \multirow{2}{*}{$\begin{array}{l}\text { Power Series } \\
\text { Method [27] } \\
\quad(\mathrm{N}=25)\end{array}$} & \multicolumn{2}{|c|}{$\mathrm{N}=25$} & \multicolumn{2}{|c|}{$\mathrm{N}=50$} \\
\hline & & & & $\begin{array}{l}\text { Present } \\
\text { Study }\end{array}$ & $\begin{array}{l}\text { Error } \\
(\%)\end{array}$ & $\begin{array}{l}\text { Present } \\
\text { Study }\end{array}$ & $\begin{array}{c}\text { Error } \\
(\%)\end{array}$ \\
\hline \multirow{5}{*}{$\begin{array}{c}\text { Simply } \\
\text { supported - } \\
\text { simply } \\
\text { supported } \\
\text { (S-S) }\end{array}$} & 1 & 9.8696 & 9.8696 & 9.8761 & 0.066 & 9.8712 & 0.016 \\
\hline & 2 & 39.4784 & 39.4785 & 39.5825 & 0.263 & 39.5044 & 0.066 \\
\hline & 3 & 88.8264 & 87.8912 & 89.3546 & 0.591 & 88.9581 & 0.148 \\
\hline & 4 & 157.9137 & - & 159.5884 & 1.049 & 158.3300 & 0.263 \\
\hline & 5 & 246.7401 & - & 250.8457 & 1.637 & 247.7577 & 0.411 \\
\hline \multirow{5}{*}{$\begin{array}{l}\text { Clamped - } \\
\text { simply } \\
\text { supported } \\
\text { (C-S) }\end{array}$} & 1 & 15.4182 & 15.4182 & 15.4300 & 0.076 & 15.4212 & 0.019 \\
\hline & 2 & 49.9649 & 49.9623 & 50.1079 & 0.285 & 50.0006 & 0.071 \\
\hline & 3 & 104.2477 & 102.3893 & 104.9033 & 0.625 & 104.4112 & 0.157 \\
\hline & 4 & 178.2697 & - & 180.2417 & 1.094 & 178.76057 & 0.275 \\
\hline & 5 & 272.0310 & - & 276.7112 & 1.691 & 273.1925 & 0.425 \\
\hline
\end{tabular}

Table 3. First five frequency parameters for uniform EB 
Table 3. First five frequency parameters for uniform EB (cont.)

\begin{tabular}{|c|c|c|c|c|c|c|c|}
\hline \multirow[b]{2}{*}{ Supported } & \multirow{2}{*}{$\frac{0}{8}$} & \multirow{2}{*}{$\begin{array}{c}\text { Exact } \\
\text { Solution } \\
{[28]}\end{array}$} & \multirow{2}{*}{$\begin{array}{l}\text { Power Series } \\
\text { Method [27] } \\
\quad(\mathrm{N}=25)\end{array}$} & \multicolumn{2}{|c|}{$\mathrm{N}=25$} & \multicolumn{2}{|c|}{$\mathrm{N}=50$} \\
\hline & & & & $\begin{array}{l}\text { Present } \\
\text { Study }\end{array}$ & $\begin{array}{c}\text { Error } \\
(\%)\end{array}$ & $\begin{array}{l}\text { Present } \\
\text { Study }\end{array}$ & $\begin{array}{c}\text { Error } \\
(\%)\end{array}$ \\
\hline \multirow{5}{*}{$\begin{array}{l}\text { Clamped - } \\
\text { clamped } \\
\text { (C-C) }\end{array}$} & 1 & 22.3733 & 22.3733 & 22.3916 & 0.082 & 22.3779 & 0.021 \\
\hline & 2 & 61.6728 & 61.6611 & 61.8622 & 0.306 & 61.7202 & 0.077 \\
\hline & 3 & 120.9034 & 112.0120 & 121.7020 & 0.656 & 121.1028 & 0.165 \\
\hline & 4 & 199.8594 & - & 202.1557 & 1.136 & 200.4317 & 0.286 \\
\hline & 5 & 298.5555 & - & 303.8512 & 1.743 & 299.8719 & 0.439 \\
\hline \multirow{5}{*}{$\begin{array}{c}\text { Clamped - } \\
\text { free } \\
\text { (C-F) }\end{array}$} & 1 & 3.5160 & 3.5160 & 3.5158 & 0.006 & 3.5160 & 0.000 \\
\hline & 2 & 22.0345 & 22.0345 & 22.0540 & 0.088 & 22.0394 & 0.022 \\
\hline & 3 & 61.6972 & 61.8060 & 61.8860 & 0.305 & 61.7444 & 0.076 \\
\hline & 4 & 120.9019 & - & 121.7006 & 0.656 & 121.1013 & 0.165 \\
\hline & 5 & 199.8595 & - & 202.1558 & 1.136 & 200.4317 & 0.285 \\
\hline
\end{tabular}

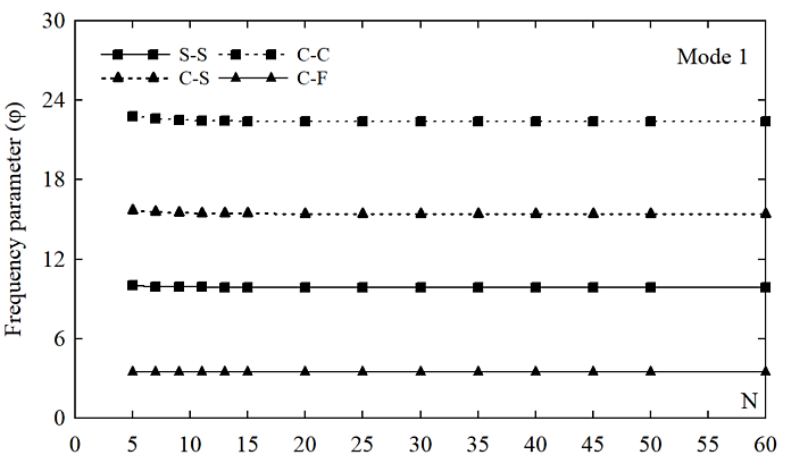

a) Mode 1

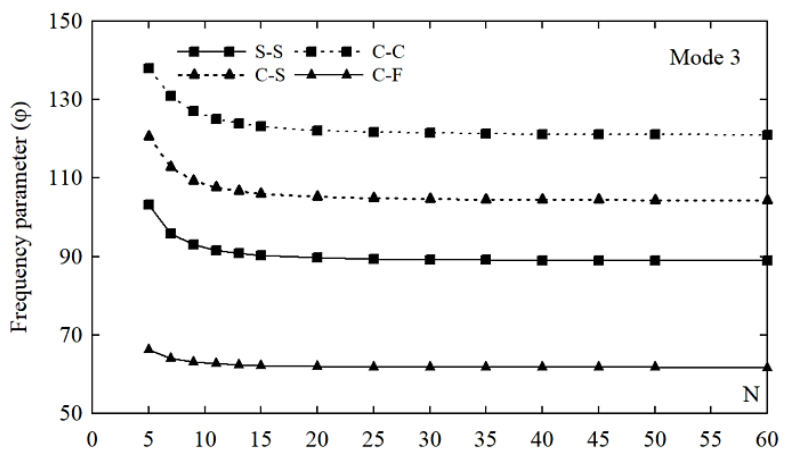

c) Mode 3

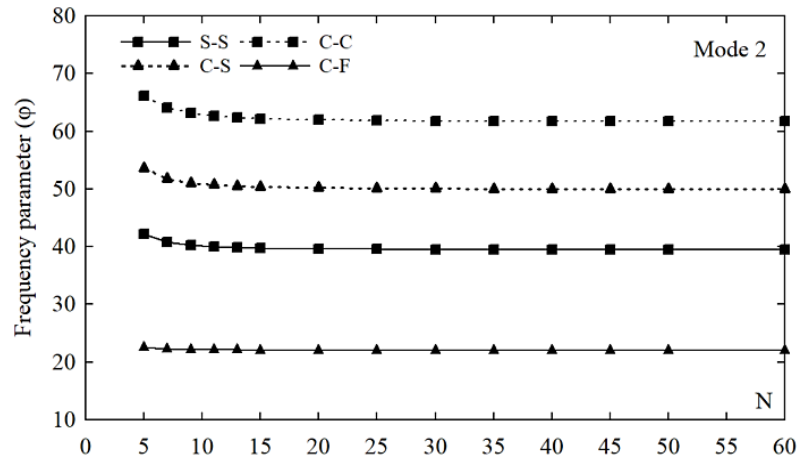

b) Mode 2

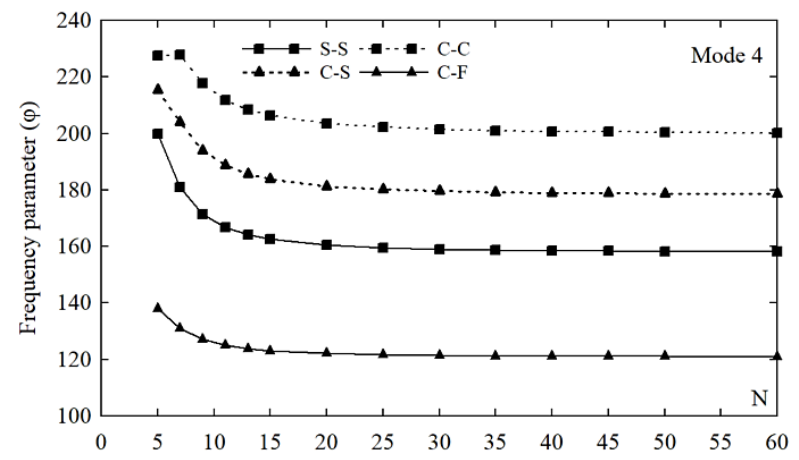

d) Mode 4

Figure 2. Convergence of frequencies parameters $(\varphi)$ of the uniform EB with $\mathrm{N}$

where $I_{0}$ and $A_{0}$ are characteristics of $\mathrm{EB}$ at the left end. Table 4 shows the frequency parameters ( $\varphi=\omega \sqrt{\rho A L^{4} / E I_{0}}$ ) of cantilever tapered EB rested on an uniform Winkler foundation using LCM [29] and this method. The calculated results using B-spline function are fairly close to LCM. 
Table 4. Frequency parameters of cantilever tapered EB on uniform foundation $(\mathrm{N}=30)$

\begin{tabular}{ccccccc}
\hline & \multicolumn{2}{c}{ Mode 1 } & \multicolumn{2}{c}{ Mode 2 } & Mode 3 \\
\cline { 2 - 6 }$K_{f}$ & LCM [29] & $\begin{array}{c}\text { Present } \\
\text { study }\end{array}$ & LCM [29] & $\begin{array}{c}\text { Present } \\
\text { study }\end{array}$ & LCM [29] & $\begin{array}{c}\text { Present } \\
\text { study }\end{array}$ \\
\hline 0 & 3.8238 & 3.8167 & 18.3173 & 18.2867 & 47.2651 & 47.2513 \\
$5 \frac{E I_{0}}{L^{2}}$ & 4.8064 & 4.8018 & 18.5214 & 18.4914 & 47.3418 & 47.3281 \\
$50 \frac{E I_{0}}{L^{2}}$ & 9.9574 & 9.9594 & 20.2721 & 20.2468 & 48.0273 & 48.0146 \\
$100 \frac{E I_{0}}{L^{2}}$ & - & 13.5333 & - & 22.0455 & - & 48.7668 \\
$500 \frac{E I_{0}}{L^{2}}$ & - & 28.7503 & - & 33.4436 & - & 54.4391 \\
$1000 \frac{E I_{0}}{L^{2}}$ & 39.5692 & 39.5729 & 44.2772 & 44.2988 & 60.8515 & 60.8569 \\
\hline
\end{tabular}

Influence of Elastically Restrained Edges on Frequency Parameter Tapered EB on Variable Winkler Foundation

As an interesting application, the influence of variable Winkler foundation and elastically restrained edges on the frequency parameter of tapered EB is evaluated. In this example, the frequency parameter of tapered EB is presented for different values of spring supported at end edges. For this purpose, the tapered EB is considered with general boundary conditions, $K_{T}$ and $K_{R}$. On the other hand, it is considered the tapered EB with linearly varying height and constant width. Also, it is assumed that EB is supported on the variable Winkler foundation (Figure 3). The EB beam characteristics are assumed as follows:

$$
\begin{gathered}
A(x)=\left\{\begin{array}{cc}
A_{0}\left(1-0.5 \frac{x}{L}\right) & x \in\left[0, \frac{L}{2}\right] \\
A_{0}\left(\frac{1}{2}+0.5 \frac{x}{L}\right) & x \in\left[\frac{L}{2}, L\right]
\end{array} \quad \begin{array}{l}
I_{0}\left(1-0.5 \frac{x}{L}\right)^{3} \\
I_{0}\left(\frac{1}{2}+0.5 \frac{x}{L}\right)^{3} \quad x \in\left[\frac{L}{2}, L\right]
\end{array}\right] \\
K_{f}(x)=50 \frac{E I_{0}}{L^{2}}(1-0.5 x) \\
K_{T L}=K_{T R}=K_{T} \quad K_{R L}=K_{R R}=K_{R}
\end{gathered}
$$

where $A_{0}$ and $I_{0}$ are cross-sectional area and second area moment of tarped EB at the right and the left ends. Figure 4 displays 2D contour graph of first frequency parameter of tapered EB on variable Winkler foundation for different spring supporting. Table 5 demonstrates the frequency parameters $\left(\varphi=\omega \sqrt{\rho A L^{4} / E I_{0}}\right)$ of the tapered EB rested on Winkler foundation with linear modulus. It is seen from results that tarped EB on variable foundation can be assumed as clamped beam when values of $K_{T} / E I_{0}$ and $K_{R} / E I_{0}$ are greater than 10000 .

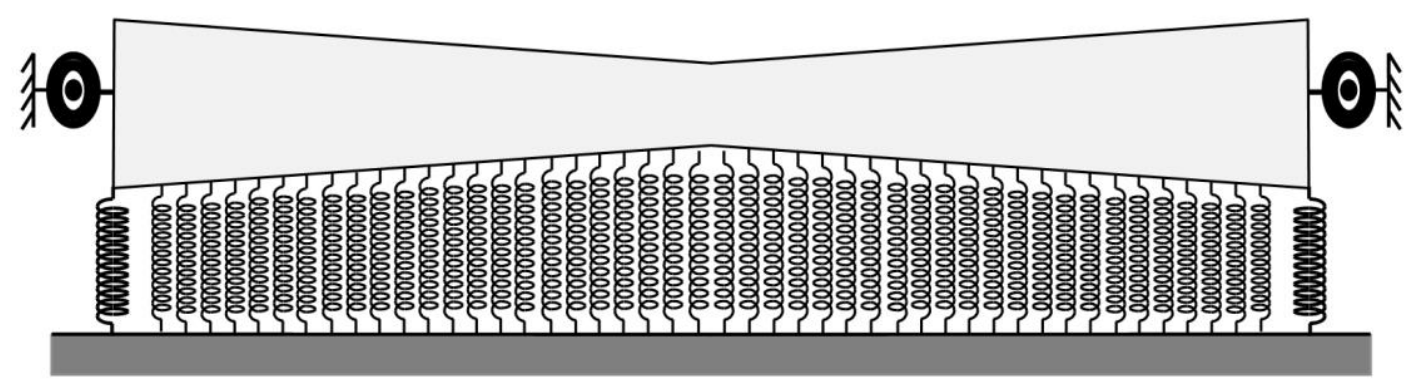

Figure 3. Tapered EB with elastic boundary conditions on variable Winkler foundation 


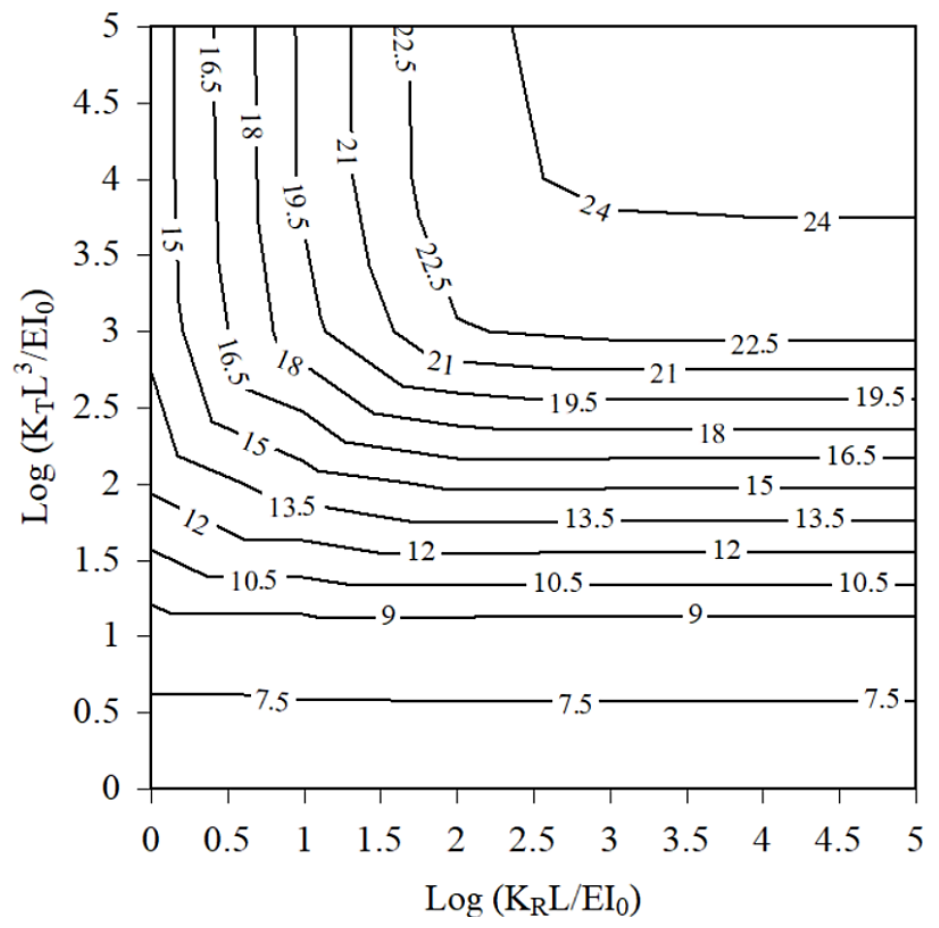

Figure 4. Variation of first frequency parameter of tapered EB on variable Winkler foundation for different spring supporting

Table 5. Frequency parameter for tapered EB with elastic boundary conditions on variable Winkler foundation $(\mathrm{N}=35)$

\begin{tabular}{|c|c|c|c|c|c|c|c|c|}
\hline \multirow{2}{*}{$K_{T} L^{3} / E I_{0}$} & \multirow{2}{*}{ Mode } & \multicolumn{7}{|c|}{$K_{R} L / E I_{0}$} \\
\hline & & 0 & 1 & 10 & 100 & 1000 & 10000 & 100000 \\
\hline \multirow[t]{3}{*}{0} & 1 & 5.6905 & 6.2348 & 6.4316 & 6.4546 & 6.4569 & 6.4571 & 6.4571 \\
\hline & 2 & 7.0497 & 7.9426 & 10.1909 & 10.9964 & 11.0977 & 11.1081 & 11.1091 \\
\hline & 3 & 20.8784 & 24.4574 & 33.2389 & 37.3793 & 37.9525 & 38.0121 & 38.0180 \\
\hline \multirow[t]{3}{*}{1} & 1 & 6.0999 & 6.4888 & 6.6318 & 6.6481 & 6.6497 & 6.6499 & 6.6499 \\
\hline & 2 & 7.3676 & 8.2772 & 10.4158 & 11.1860 & 11.2830 & 11.2930 & 11.2940 \\
\hline & 3 & 21.0554 & 24.5980 & 33.3126 & 37.4298 & 38.0001 & 38.0594 & 38.0653 \\
\hline \multirow[t]{3}{*}{10} & 1 & 8.1181 & 8.1320 & 8.1414 & 8.1427 & 8.1428 & 8.1428 & 8.1428 \\
\hline & 2 & 10.1862 & 10.8595 & 12.2345 & 12.7548 & 12.8215 & 12.8284 & 12.8291 \\
\hline & 3 & 22.6731 & 25.8746 & 33.9811 & 37.8878 & 38.4322 & 38.4888 & 38.4945 \\
\hline \multirow[t]{3}{*}{100} & 1 & 11.4107 & 12.3026 & 14.3031 & 15.3121 & 15.2414 & 15.2527 & 15.2538 \\
\hline & 2 & 22.4807 & 22.4978 & 22.5407 & 22.5606 & 22.5634 & 22.5637 & 22.5637 \\
\hline & 3 & 36.1380 & 37.2213 & 40.6551 & 42.6753 & 42.9767 & 43.0084 & 43.0115 \\
\hline \multirow[t]{3}{*}{1000} & 1 & 12.3567 & 13.9381 & 19.0201 & 22.3865 & 22.9319 & 22.9898 & 22.9957 \\
\hline & 2 & 33.1822 & 34.6709 & 40.5439 & 45.4176 & 46.2910 & 46.3853 & 46.3948 \\
\hline & 3 & 69.5546 & 70.0591 & 72.2519 & 74.2917 & 74.6755 & 74.7173 & 74.7215 \\
\hline \multirow[t]{3}{*}{10000} & 1 & 12.4638 & 14.1362 & 19.7325 & 23.6331 & 24.2779 & 24.3466 & 24.3535 \\
\hline & 2 & 34.7832 & 36.7397 & 45.2571 & 53.4607 & 55.0463 & 55.2196 & 55.2371 \\
\hline & 3 & 79.3806 & 81.1417 & 90.5526 & 102.895 & 105.735 & 106.055 & 106.088 \\
\hline \multirow[t]{3}{*}{100000} & 1 & 12.4746 & 14.1564 & 19.8061 & 23.7617 & 24.4164 & 24.4862 & 24.4932 \\
\hline & 2 & 34.9447 & 36.9507 & 45.7556 & 54.3116 & 55.9670 & 56.1478 & 56.1661 \\
\hline & 3 & 80.3417 & 82.2578 & 92.6405 & 106.440 & 109.602 & 109.958 & 109.994 \\
\hline
\end{tabular}


From results are shown in Table 5, it illustrates that in the tapered EB with $K_{T} L^{3} / E I_{0}=K_{R} L / E I_{0}=10000,1^{\text {st }}, 2^{\text {nd }}$ and $3^{\text {rd }}$ frequency parameter are $24.3466,55.2196$ and 106.055, respectively. In comparison with the tapered EB with $K_{T} L^{3} / E I_{0}=K_{R} L / E I_{0}=10000$, maximum difference of $1^{\text {st }}, 2^{\text {nd }}$ and $3^{\text {rd }}$ frequency parameter for tapered EB with $K_{T} L^{3} / E I_{0}=10000$ and $K_{R} L / E I_{0}=0$ are approximately $48.81 \%, 37.01 \%$ and $25.15 \%$, respectively. Also, it illustrates that values of $1^{\text {st }}$ frequency parameters of tapered EB on variable Winkler foundation are almost the same when the $K_{R} L / E I_{0}$ is larger than 100 .

\section{Double Tapered EB on Variable Winkler Foundation}

As an interesting application, the influence of the double taper of the beam shape on the frequency parameter is evaluated. In this example, it is assumed that EB is supported on the variable Winkler foundation (Figure 5). The EB beam characteristics are assumed as follows:

$$
\begin{gathered}
A(x)=A_{0}\left(1-\alpha \frac{x}{L}\right)^{2} \quad I(x)=I_{0}\left(1-\alpha \frac{x}{L}\right)^{4} \\
K_{f}(x)=50 \frac{E I_{0}}{L^{2}}(1-x) \quad \xi_{e}=0.10 \quad \xi_{i}=0 \quad L_{1}-L_{2}=L
\end{gathered}
$$

where $I_{0}$ and $A_{0}$ are the characteristics of the EB at the left end. Table 6 demonstrates the frequency parameters $\left(\varphi=\omega \sqrt{\rho A L^{4} / E I_{0}}\right)$ of the double tapered EB on Winkler foundation with linear modulus for different values of $\alpha$.

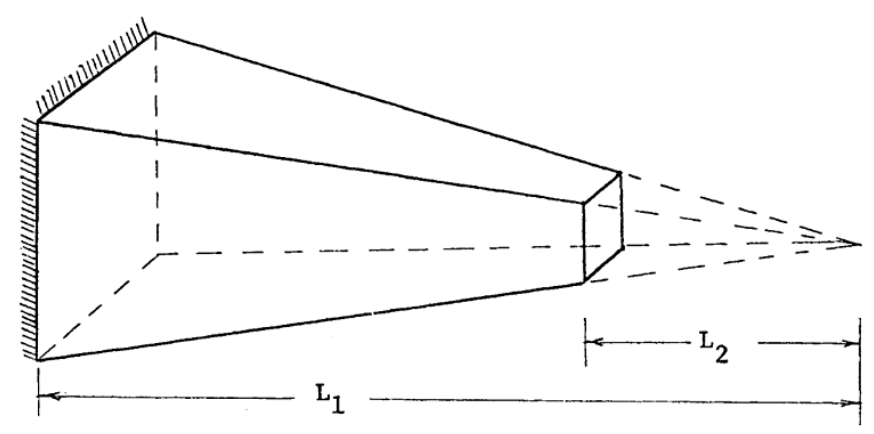

Figure 5. Double tapered EB with elastic boundary conditions on variable Winkler foundation

Table 6. Frequency parameter of tapered EB with elastic boundary conditions on variable Winkler foundation $(\mathrm{N}=30)$

\begin{tabular}{ccccc}
\hline Mode & $\alpha=0$ & $\alpha=0.25$ & $\alpha=0.50$ & $\alpha=0.75$ \\
\hline 1 & 4.6556 & 5.4434 & 6.7167 & 9.2514 \\
2 & 22.3924 & 21.2963 & 20.2310 & 19.5247 \\
3 & 61.7062 & 55.3777 & 48.6353 & 41.2963 \\
4 & 120.9467 & 106.8945 & 91.6538 & 74.2248 \\
5 & 200.5029 & 176.0969 & 149.4431 & 118.4254 \\
\hline
\end{tabular}

\section{CONCLUSION}

This paper introduces a numerical solution approach of free vibration analysis of tapered damped EB rested on a variable Winkler foundation. For this proposed, quintic B-spline collocation method is applied to solve governing differential equation. The presented method yields the semi-closed solutions. Therefore, it is more efficient for complex systems. Also, the tapered EB with general boundary conditions can be embedded in this method. The numerical examples are presented to show efficiency and applicability of the presented method. Finally, it efficiency and reliability of the quintic B-spline collocation method are demonstrated from obtained results. Therefore, it can be seen that algorithm converge is increased with the number of terms. 


\section{ACKNOWLEDGMENTS}

This study was supported by University of Mohaghegh Ardabili (Ref. No.: 89).

\section{REFERENCES}

[1] A. Ghannadiasl and M. Mofid, "An analytical solution for free vibration of elastically restrained Timoshenko beam on an arbitrary variable Winkler foundation and under axial load," Latin American Journal of Solids and Structures, an ABCM Journal, vol. 12, no. 13, pp. 2417-2438, 2015, doi: 10.1590/1679-78251504.

[2] S. Binesh, "Analysis of beam on elastic foundation using the radial point interpolation method," Scientia Iranica, vol. 19, no. 3, pp. 403-409, 2012, doi: 10.1016/j.scient.2012.04.003.

[3] N. N. A. Hamid, A. A. Majid, and A. I. M. Ismail, "Quartic B-spline interpolation method for linear two-point boundary value problem," World Applied Sciences Journal, vol. 17, pp. 39-43, 2012.

[4] B. Bialecki and G. Fairweather, "Orthogonal spline collocation methods for partial differential equations," Journal of Computational and Applied Mathematics, vol. 128, no. 1-2, pp. 55-82, 2001, doi: 10.1016/S0377-0427(00)00509-4.

[5] J. Rashidinia, R. Jalilian, R. Mohammadi, and M. Ghasemi, "Sextic spline method for the solution of a system of obstacle problems," Applied mathematics and computation, vol. 190, no. 2, pp. 1669-1674, 2007, doi: 10.1016/j.amc.2007.02.044.

[6] B. Saka and İ. Dağ, "Quartic B-spline collocation method to the numerical solutions of the Burgers' equation," Chaos, Solitons \& Fractals, vol. 32, no. 3, pp. 1125-1137, 2007, doi: 10.1016/j.chaos.2005.11.037.

[7] M. K. Kadalbajoo and A. S. Yadaw, "B-Spline collocation method for a two-parameter singularly perturbed convectiondiffusion boundary value problems," Applied Mathematics and Computation, vol. 201, no. 1-2, pp. 504-513, 2008, doi: 10.1016/j.amc.2007.12.038.

[8] M. Ramadan, I. Lashien, and W. Zahra, "Quintic nonpolynomial spline solutions for fourth order two-point boundary value problem," Communications in Nonlinear Science and Numerical Simulation, vol. 14, no. 4, pp. 1105-1114, 2009, doi: 10.1016/j.cnsns.2007.12.008.

[9] M.-H. Hsu, "Vibration analysis of non-uniform beams resting on elastic foundations using the spline collocation method," Tamkang Journal of Science and Engineering, vol. 12, no. 2, pp. 113-122, 2009, doi: 10.6180/jase.2009.12.2.02.

[10] I. Aziz and B. Šarler, "The numerical solution of second-order boundary-value problems by collocation method with the Haar wavelets," Mathematical and Computer Modelling, vol. 52, no. 9-10, pp. 1577-1590, 2010, doi: 10.1016/j.mcm.2010.06.023.

[11] L. B. Da Veiga, C. Lovadina, and A. Reali, "Avoiding shear locking for the Timoshenko beam problem via isogeometric collocation methods," Computer Methods in Applied Mechanics and Engineering, vol. 241, pp. 38-51, 2012, doi: 10.1016/j.cma.2012.05.020.

[12] M. Zarebnia and R. Parvaz, "Septic B-spline collocation method for numerical solution of the Kuramoto-Sivashinsky equation," International Journal of Mathematical and Computational Sciences, vol. 7, no. 3, pp. 544-548, 2013, doi: 10.5281/zenodo.1088240.

[13] M. Zarebnia and R. Parvaz, "B-spline collocation method for numerical solution of the nonlinear two-point boundary value problems with applications to chemical reactor theory," International Journal of Mathematical Engineering and Science, vol. 3, no. 3, pp. 6-10, 2014.

[14] R. Mohammadi, "Sextic B-spline collocation method for solving Euler-Bernoulli Beam Models," Applied Mathematics and Computation, vol. 241, pp. 151-166, 2014, doi: 10.1016/j.amc.2014.05.008.

[15] A. Reali and H. Gomez, "An isogeometric collocation approach for Bernoulli-Euler beams and Kirchhoff plates," Computer Methods in Applied Mechanics and Engineering, vol. 284, pp. 623-636, 2015, doi: 10.1016/j.cma.2014.10.027.

[16] J. Kiendl, F. Auricchio, L. B. da Veiga, C. Lovadina, and A. Reali, "Isogeometric collocation methods for the Reissner-Mindlin plate problem," Computer Methods in Applied Mechanics and Engineering, vol. 284, pp. 489-507, 2015, doi: 10.1016/j.cma.2014.09.011.

[17] G. Akram, "Solution of the system of fifth order boundary value problem using sextic spline," Journal of the Egyptian Mathematical Society, vol. 23, no. 2, pp. 406-409, 2015, doi: 10.1016/j.joems.2014.04.009.

[18] F. Auricchio, L. B. da Veiga, J. Kiendl, C. Lovadina, and A. Reali, "Isogeometric collocation mixed methods for rods," Discrete \& Continuous Dynamical Systems-S, vol. 9, no. 1, p. 33, 2016, doi: 10.3934/dcdss.2016.9.33.

[19] C.-S. Liu and B. Li, "Forced and free vibrations of composite beams solved by an energetic boundary functions collocation method," Mathematics and Computers in Simulation, vol. 177, pp. 152-168, 2020, doi: j.matcom.2020.04.020.

[20] Q. Ren and H. Tian, "Numerical solution of the static beam problem by Bernoulli collocation method," Applied Mathematical Modelling, vol. 40, no. 21-22, pp. 8886-8897, 2016, doi: 10.1016/j.apm.2016.05.018.

[21] İ. Çelik, "Free vibration of non-uniform Euler-Bernoulli beam under various supporting conditions using Chebyshev wavelet collocation method," Applied Mathematical Modelling, vol. 54, pp. 268-280, 2018, doi: 10.1016/j.apm.2017.09.041.

[22] J. Kiendl, F. Auricchio, and A. Reali, "A displacement-free formulation for the Timoshenko beam problem and a corresponding isogeometric collocation approach," Meccanica, vol. 53, no. 6, pp. 1403-1413, 2018, doi: 10.1007/s11012-017-0745-7.

[23] W.-R. Chen, "Vibration Analysis of Axially Functionally Graded Tapered Euler-Bernoulli Beams Based on Chebyshev Collocation Method," International Journal of Acoustics and Vibration, vol. 25, no. 3, pp. 436-444, 2020, doi: 10.20855/ijav.2020.25.31680. 
[24] J. Prochazkova, "Derivative of B-Spline function," in Proceedings of the 25th Conference on Geometry and Computer Graphics, Prague, Czech Republic, 2005, pp. 12-16.

[25] M. A. Hilal and H. S. Zibdeh, "Vibration analysis of beams with general boundary conditions traversed by a moving force," Journal of Sound and Vibration, vol. 229, no. 2, pp. 377-388, 2000, doi: 10.1006/jsvi.1999.2491.

[26] A. Ghannadiasl, "Analytical Study of Dynamic Response of Railway on Partial Elastic Foundation under Travelling Accelerating Concentrated Load," International Journal of Transportation Engineering, vol. 4, no. 4, pp. 317-334, 2017, doi: 10.22119/IJTE.2017.44434.

[27] A. Ghannadiasl, "Natural frequencies of the elastically end restrained non-uniform Timoshenko beam using the power series method," Mechanics Based Design of Structures and Machines, vol. 47, no. 2, pp. 201-214, 2019, doi: 10.1080/15397734.2018.1526691.

[28] C. Y. Wang and C. Wang, Structural vibration: exact solutions for strings, membranes, beams, and plates. CRC Press, 2019.

[29] A. Ghannadiasl, A. Zamiri, and A. Borhanifar, "Free vibrations of non-uniform beams on a non-uniform Winkler foundation using the Laguerre collocation method," Journal of the Brazilian Society of Mechanical Sciences and Engineering, vol. 42, no. 5, pp. 1-12, 2020, doi: 10.1007/s40430-020-02332-3. 